\title{
CENTRALIZERS OF $p$-SUBGROUPS IN SIMPLE LOCALLY FINITE GROUPS
}

\author{
KIVANÇ ERSOY \\ Institute of Mathematics, Freie Universität Berlin, 14195 Berlin, Germany and \\ Department of Mathematics, Mimar Sinan Fine Arts University, Istanbul 34427, Turkey \\ e-mail: ersoykivanc@gmail.com
}

(Received 17 May 2018; revised 17 December 2018; accepted 7 January 2019; first published online 21 February 2019)

\begin{abstract}
In Ersoy et al. [J. Algebra 481 (2017), 1-11], we have proved that if $G$ is a locally finite group with an elementary abelian $p$-subgroup $A$ of order strictly greater than $p^{2}$ such that $C_{G}(A)$ is Chernikov and for every non-identity $\alpha \in A$ the centralizer $C_{G}(\alpha)$ does not involve an infinite simple group, then $G$ is almost locally soluble. This result is a consequence of another result proved in Ersoy et al. [J. Algebra 481 (2017), 1-11], namely: if $G$ is a simple locally finite group with an elementary abelian group $A$ of automorphisms acting on it such that the order of $A$ is greater than $p^{2}$, the centralizer $C_{G}(A)$ is Chernikov and for every non-identity $\alpha \in A$, the set of fixed points $C_{G}(\alpha)$ does not involve an infinite simple groups then $G$ is finite. In this paper, we improve this result about simple locally finite groups: Indeed, suppose that $G$ is a simple locally finite group, consider a finite nonabelian subgroup $P$ of automorphisms of exponent $p$ such that the centralizer $C_{G}(P)$ is Chernikov and for every non-identity $\alpha \in P$ the set of fixed points $C_{G}(\alpha)$ does not involve an infinite simple group. We prove that if $\operatorname{Aut}(G)$ has such a subgroup, then $G \cong P \operatorname{PL}_{p}(k)$ where char $k \neq p$ and $P$ has a subgroup $Q$ of order $p^{2}$ such that $C_{G}(P)=Q$.
\end{abstract}

2010 Mathematics Subject Classification. Primary 20E32, 20E36; Secondary 20G15

1. Introduction. In [2], we have proved the following result:

THEOREM 1.1. [2, Theorem 1.1]. Let p be a prime and G a locally finite group containing an elementary abelian p-subgroup A of rank at least 3 such that $C_{G}(A)$ is Chernikov and $C_{G}(a)$ involves no infinite simple groups for any $a \in A^{\#}$. Then $G$ is almost locally soluble.

To prove Theorem 1.1, we gave the following characterization of $P S L_{p}(k)$ where chark $\neq p$.

THEOREM 1.2. [2, Theorem 1.2]. An infinite simple locally finite group $G$ admits an elementary abelian p-group of automorphisms $A$ such that $C_{G}(A)$ is Chernikov and $C_{G}(a)$ involves no infinite simple groups for any $a \in A^{\#}$ if and only if $G$ is isomorphic to $\operatorname{PSL}_{p}(k)$ for some locally finite field $k$ of characteristic different from $p$ and $A$ has order $p^{2}$.

In this paper, we will improve Theorem 1.2. Indeed, we will prove a similar result without assuming $A$ is an elementary abelian, but instead, we prove for any subgroup of exponent $p$.

THEOREM 1.3. Let $G$ be an infinite simple locally finite group, $P$ a subgroup of automorphisms of exponent $p$ such that

(1) $C_{G}(P)$ is Chernikov, 
(2) For every $\alpha \in P \backslash\{1\}$, the set of fixed points $C_{G}(\alpha)$ does not involve an infinite simple group.

Then $G \cong P S L_{p}(k)$ where $k$ is an infinite locally finite field of characteristic $p$ and $P$ has a subgroup $Q$ of order $p^{2}$ such that $C_{G}(P)=C_{G}(Q)=Q$.

2. Preliminaries. Let us recall some definitions of the concepts mentioned in the theorems. First, consider $C_{p^{n}}=\left\{x \in \mathbb{C}: x^{p^{n}}=1\right\}$. Here, $\left(C_{p^{n}},.\right)$ defines a group isomorphic to a cyclic group of order $p^{n}$. Observe that if $m \mid n$ then $C_{p^{m}} \leq C_{p^{n}}$, and with the inclusion maps, these sets form a direct system, where the direct limit

$$
\lim _{n \in \mathbb{N}} C_{p^{n}}
$$

is denoted by $C_{p^{\infty}}$, which consists of all complex $p^{n}$-th roots of unity, and forms a group under complex multiplication. This group is called the quasi-cylic $p$-group.

Definition 2.1. A group is called a Chernikov group if it is a finite extension of a direct product of finitely many copies of some quasi-cyclic $p_{i}$-groups, for possibly distinct primes $p_{i}$.

DEFINITION 2.2. Let $\chi$ be a group-theoretical property. If a group $G$ has a normal subgroup of finite index satisfying $\chi$, then $G$ is called almost $\chi$.

Definition 2.3. Let $G$ and $H$ be two groups. If $G$ has a normal subgroup $K$ such that $G / K$ has a subgroup isomorphic to $H$, then $G$ is said to involve a subgroup isomorphic to $H$.

Definition 2.4. A group satisfies the minimal condition, namely min, if any non-empty set of subgroups has a minimal subgroup. A group satisfies min- $p$ if any non-empty set of $p$-subgroups has a minimal subgroup.

Kegel-Wehrfritz and Sunkov proved independently that a locally finite group satisfying minimal condition is a Chernikov group (see $[5,9])$. For detailed discussion of groups satisfying $\min$ and $\min -p$, see [6].

3. Main results. First, we need the following proposition:

Proposition 3.1. Let $\bar{G}$ be a simple linear algebraic group of adjoint type over the algebraic closure of $\mathbb{F}_{q}$, let $g \in \bar{G}$ be an element of prime order $p \neq q$ such that $C_{\bar{G}}(g)$ is a non-abelian group which does not involve any infinite simple groups. Then

(i) The identity component $C_{\bar{G}}(g)^{0}$ of the centralizer of $g$ in $\bar{G}$ is a maximal torus of $\bar{G}$,

(ii) $\bar{G} \cong P G L_{p}\left(\overline{\mathbb{F}_{q}}\right)$.

Proof. Since $\bar{G}$ is a simple linear algebraic group of adjoint type over the algebraic closure of $\mathbb{F}_{q}$ and $g \in G$ a semisimple element, $g$ is contained in a maximal torus $T$ of $\bar{G}$. By [7, Propositions 14.1 and 14.2], $C_{\bar{G}}(g)^{0}$ is connected reductive, containing a maximal torus $T$, and involving no infinite simple groups. Hence, $C_{\bar{G}}(g)^{0}=T$. By [7, Proposition 14.20], the exponent of $C_{\bar{G}}(g) / C_{\bar{G}}(g)^{0}$ divides $p$, hence either $C_{\bar{G}}(g)$ is connected, and hence a torus, or $C_{\bar{G}}(g) / C_{\bar{G}}(g)^{0}$ is a finite group of exponent $p$.

Since $C_{\bar{G}}(g)$ is not abelian, one has $C_{\bar{G}}(g)$ a finite extension of an abelian group $T$, so it has finite rank. Recall that an infinite group $G$ is said to have finite rank $r$ if every finitely 
generated subgroup is $r$-generated. In [1, Theorem 1.8], we have shown that when a simple linear algebraic group $\bar{G}$ over the algebraic closure of $\mathbb{F}_{q}$ has an element $g$ of order $p$ with $C_{\bar{G}}(z)$ has finite rank, then one of the following cases occur:

(1) $\bar{G}$ is of type $A_{l}$ and $p>l$,

(2) $\bar{G}$ is of type $B_{l}, C_{l}$, and $p>2 l-1$,

(3) $\bar{G}$ has type $D_{l}$ and $p>2 l-3$,

(4) $\bar{G}$ is isomorphic to one of $E_{6}, E_{7}, E_{8}, F_{4}$, or $G_{2}$ and $p>11,17,29,17$, or 5 , respectively.

On the other hand, since $C_{\bar{G}}(g) / C_{\bar{G}}(g)^{0}$ has exponent $p$, by [10, Corollary 4.4] and [7, Proposition 14.20], we get $p$ is a torsion prime. The list of torsion primes of linear algebraic groups is defined as follows: for type $A_{l}$, these are the primes that divide $l+1$. For types $B_{l}, C_{l}, D_{l}, G_{2}$, the prime is 2 . For types $E_{6}, E_{7}, F_{4}$, the primes are 2 and 3 , and for type $E_{8}$, the primes are 2, 3, 5 (see [8]).

Hence, one deduce that the only possible case that may occur is $\bar{G}$ has type $A_{p-1}$, indeed $\bar{G} \cong P G L_{p}\left(\overline{\mathbb{F}}_{q}\right)$.

THEOREM 3.2. Let $G$ be an infinite simple locally finite group with a finite non-abelian p-group of automorphisms $P$ such that

(1) $C_{G}(P)$ is Chernikov,

(2) For every $\alpha \in P \backslash\{1\}$, the set of fixed points $C_{G}(\alpha)$ does not involve an infinite simple group

Then, $G$ is isomorphic to $P S L_{p}(k)$ where $k$ is a locally finite field of characteristic $q \neq p$ and $P$ is metabelian.

Proof. Since $P$ is a finite $p$-group and $C_{G}(P)$ satisfies min-p, by [2, Lemma 2.1], $G$ satisfies $\min -p$. Then, by [4, Theorem B], $G$ is a simple group of Lie type over a locally finite field $k$ of characteristic $q$. Now assume that $q=p$. Clearly $G$ contains a root subgroup, which is an infinite elementary abelian $p$-subgroup. Hence, $G$ can not satisfy min- $p$. Hence, $q \neq p$, that is, $G$ is isomorphic to a simple group of Lie type over an infinite locally finite field of characteristic $q \neq p$.

Now, by [3, Lemma 4.3], there exists a simple linear algebraic group $\bar{G}$ of adjoint type, a Frobenius map $\sigma$ on $\bar{G}$ and a sequence of natural numbers $n_{i} \mid n_{i+1}$ such that

$$
G=\bigcup_{i \in \mathbb{N}} O^{p^{\prime}}\left(\bar{G}_{\sigma^{n_{i}}}\right)
$$

By assumption, the centralizer of any non-identity element does not involve an infinite simple group, so [2, Lemma 2.3] implies that $P$ consists of inner-diagonal automorphisms of $G$. Hence, $P \leq \bigcup_{i \in \mathbb{N}} \bar{G}_{\sigma^{n_{i}}}$. Therefore, $P \leq \bar{G}_{\sigma^{n_{j}}}$ for some $j \in \mathbb{N}$.

Choose $1 \neq z \in Z(P)$. Clearly, $P \leq C_{\bar{G}}(z)$. Now, $C_{G}(z)=\bigcup_{i \in \mathbb{N}} O^{p^{\prime}}\left(C_{\bar{G}}(z)_{\sigma^{n_{i}}}\right)$.

By assumption, $C_{G}(z)$ does not involve an infinite simple group. Now, suppose that $C_{\bar{G}}(z)$ involves a simple linear algebraic group $H$. Consider the union of fixed points of $\sigma^{n_{i}}$ on $H$, denoted by $H_{i}=H_{\sigma^{n_{i}}}$. Clearly, $H_{i} \leq H_{i+1}$ and infinitely many of $H$ involves finite simple groups such that their union form an infinite locally finite simple group. Hence, we get a contradiction and we deduce $C_{\bar{G}}(z)$ does not involve a simple linear algebraic group. By [2, Lemma 2.4], $C_{\bar{G}}(z)$ is metabelian. Hence, $P$ is metabelian. On the other hand, since $P$ is not abelian, $C_{\bar{G}}(z)$ is not abelian.

By Proposition 3.1, $\bar{G}$ is isomorphic to $P G L_{p}\left(\overline{\mathbb{F}_{q}}\right)$. Hence, $G$ is isomorphic to either $\operatorname{PSL}_{p}(k)$ or $P S U_{p}(k)$. Following the argument in the proof of Theorem 1.2 in [2], since 
the Weyl group of $P S U_{p}(k)$ has no elements of order $p$, and $P T / T$ embeds in the Weyl group, $P S U_{p}(k)$ has no such non-abelian subgroup $P$. Therefore, $G \cong P S L_{p}(k)$ where $k$ is an infinite locally finite field of characteristic $q \neq p$.

Then, we prove the main result of the paper:

Proof of Theorem 1.3. Assume first that $P$ is abelian. Then by Theorem 1.2, the result follows with $|P|=p^{2}$.

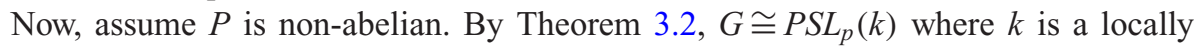
finite field of characteristic $q \neq p$. Let $1 \neq z \in Z(P)$, observe that $P \leq C_{G}(z) \leq C_{\bar{G}}(z)$ where $\bar{G}$ is the corresponding simple linear algebraic group and $\sigma$ is the Frobenius map such that $G=\bigcup_{i \in \mathbb{N}} O^{p^{\prime}}\left(\bar{G}_{\sigma^{n_{i}}}\right)$, which exist by [3, Lemma 4.3]. Denote the maximal torus of $\bar{G}$ containing $z$ by $T$. By Proposition 3.1(i), $C_{\bar{G}}(z)^{0}=T$. Indeed, by [10, Corollary 1.7], $T$ is the unique maximal torus containing $z$. Since $P$ is not abelian, $C_{\bar{G}}(z) / C_{\bar{G}}(z)^{0}$ can not be 1 , hence by [7, Proposition 14.20], it has exponent $p$. Let $y$ be any element of $C_{\bar{G}}(z) \backslash C_{\bar{G}}(z)^{0}$. Then, $Q=\langle y, z\rangle$ has order $p^{2}$. Indeed, $C_{\bar{G}}(z)^{0}=T$, and $y \in N_{\bar{G}}(T)$. Hence, $y$ induces an element $w$ of order $p$ in the Weyl group. Now, $z \in C_{T}(w)$. The computation in the proof of Theorem 1.2 in [2] shows that indeed $C_{T}(w)$ has order $p$, hence $C_{\bar{G}}(Q)=Q$. This $Q$ is the required subgroup.

ACKNOWLEDGEMENTS. This research was carried out when the author was visiting EPFL and TU Kaiserslautern. The author thanks Professor Donna Testerman and Professor Gunter Malle for their hospitality. The author was partially supported by Mimar Sinan Fine Arts University Research Project Unit, with project number 2017/21. The author thanks the referee for the suggestions and comments, which were helpful to improve the text.

\section{REFERENCES}

1. K. Ersoy and C. K. Gupta, Locally finite groups with centralizers of finite rank, Comm. Alg. 44(12) (2016), 5074-5087.

2. K. Ersoy, M. Kuzucuoğlu and P. Shumyatsky, Locally finite groups and their subgroups with small centralizers, J. Algebra 481 (2017), 1-11.

3. B. Hartley and M. Kuzucuoğlu, Centralizers of elements in locally finite simple groups, Proc. London Math. Soc. 62 (1991), 301-324.

4. B. Hartley and G. Shute, Monomorphisms and direct limits of finite groups of Lie type, Q. J. Math. 35(1) (1984), 49-71

5. O. H. Kegel and B. A. F. Wehrfritz, Strong finiteness conditions in locally finite groups, Math. Z. 117 (1970), 309-324. 1973).

6. O. H. Kegel and B. A. F. Wehrfritz, Locally finite groups (North-Holland, Amsterdam,

7. G. Malle and D. Testerman, Linear algebraic groups and finite groups of Lie type, Cambridge Studies in Advanced Mathematics, vol. 133 (Cambridge University Press, Cambridge, 2011).

8. R. Steinberg, Torsion in reductive groups, Adv. Math. 15(1) (1975), 63-92.

9. V. P. Shunkov, On the minimality problem for locally finite groups, Algebra Logic 9 (1970), $137-151$

10. T. A. Springer and R. Steinberg, "Conjugacy Classes", in Seminar on Algebraic Groups and Related Finite Groups, Lecture Notes in Mathematics, vol. 131 (Springer-Verlag, Berlin, 1970). 